\title{
Large Deflection of Functionally Graded Porous Beams Based on a Geometrically Exact Theory with a Fully Intrinsic Formulation
}

\author{
Pedram Khaneh Masjedi ${ }^{\mathrm{a}, *}$, Alireza Maheri $^{\mathrm{b}}$, Paul M. Weaver ${ }^{\mathrm{a}}$ \\ ${ }^{a}$ Bernal Institute, School of Engineering, University of Limerick, Limerick, Ireland \\ ${ }^{b}$ School of Engineering, University of Aberdeen, Aberdeen, UK
}

\begin{abstract}
Porous graded materials found in nature can be regarded as variable stiffness optimized load carrier elements that exhibit beneficial properties for real-life engineering designs. In order to investigate the nonlinear behaviour of variable stiffness bioinspired materials, the large deflection of functionally graded beams made from porous materials is considered in this work. Our purpose is to present an efficient and accurate methodology capable of capturing spatially large deflections of these structures with different types of loading conditions and porosity distributions. A geometrically exact beam model with fully intrinsic formulation is employed for the first time to study the large deflection behaviour of functionally graded beams under conservative and non-conservative (follower) loading scenarios. An orthogonal Chebyshev collocation method is used for the discretisation of the fully intrinsic formulation. Two types of porosity distributions, namely cross-sectional and span-wise, are considered and the effect of porosity distribution has been investigated for various benchmark classical test cases. It is shown that to obtain a given level of accuracy, the span-wise functionally graded beam is computationally more demanding compared to the cross-sectional functionally graded beam. In addition to classical problems, two examples demonstrating 3D deflections of a highly flexible structures made from porous material subject to combined loads are investigated. It is shown that the current paradigm, while being computationally efficient, can effectively capture the large deflections of functionally graded beams with excellent accuracy.
\end{abstract}

Keywords: Bioinspired Materials, Functionally Graded Beams, Porous Materials, Geometrically Exact Beam, Large deflection, Intrinsic Formulation

\section{Introduction}

Bioinspired materials play a key role in recent advances in technology. These materials satisfy multiple design criteria due to their variable and state-dependent characteristics (Saavedra Flores et al. [1]) and therefore circumvent the usual trade-offs between shape adaptability, load-bearing capability and stability.

\footnotetext{
*Corresponding author: Phone:+353 834603273

Email address: pedram.masjedi@ul.ie; pk.masjedi@gmail.com (Pedram Khaneh Masjedi)
} 
Applications include plant biomechanics (Rafsanjani et al. [2], Zhao et al. [3], Gonzalez and Nguyen [4]), bone biomechanics (Bottlang and Long [5], Heyland et al. [6]), biological inspired sensing systems (Birdwell et al. [7, Li et al. [8, Mongeau et al. 9]) and soft robotics (Trivedi et al. [10, Mac Murray et al. [1]).

Mechanical/structural components made of bioinspired materials, such as porous and poroelastic materials, may undergo large deflections as part of their design needs. Examples include morphing aerostructures, soft robotic arms, highly flexible and lightweight aircraft wings and wind turbine blades. Many of these components can be modelled as a functionally graded beam.

Functionally graded materials (FGM) are characterized as inhomogeneous materials in which their properties such as density and elastic modulus vary continuously over volume. Porous materials with varying porosity are functionally graded (FG) structures which are mostly found in biological materials such as human/animal bones, plant stems, etc., and act as load carriers with optimized characteristics. The porous FG structures offer a wide range of multifunctional properties including enhanced acoustics, shock resistance and heat transfer capabilities and high-performance to weight ratio. With the advance of manufacturing methods such as additive 3D printing, the manufacture of the porous FG structures is now possible (e.g. see Parthasarathy et al. [12]). All of these properties can be employed to design structures with tailored variable stiffness properties and increased manufacturability. Thus, from an engineering point of view, this natural constructional paradigm creates promising options for applying the concept of variable stiffness porous functionally graded structures for engineering applications in which optimum performance with minimal weight is sought such as aircraft wings, wind turbine rotor blades and robotic arms.

There have been a number of studies on the large deflection analysis of functionally graded structures. Kang and Li 13 investigated the large deflections of cantilever beams subject to a conservative end force and in a later work (Kang and Li [14]) subject to an end moment. Rahimi and Davoodinik [15] considered the large deflections of FG beams under inclined tip load using Adomian Decomposition Method. Kocatürk et al. [16] studied the large displacements of FG beams under non-follower (conservative) transverse uniformly distributed load by using the total Lagrangian Timoshenko beam element. Almeida et al. 17] presented the in-plane large deflections of FG beams by means of a tailored total Lagrangian formulation. Zhang 18 studied the nonlinear bending of FG beams using a high-order shear deformation theory with von Kármán strain-displacement relationships. Nguyen [19, 20, Nguyen and Gan 21] have considered the large displacement behaviour of tapered FG beams using Euler-Bernoulli or Timoshenko hypotheses, using a corotational finite element method. Sitar et al. 22] studied the large deflection of a cantilever FG beam subject to a combined loading consisting of the distributed continuous loads and point loads at the free end. Niknam et al. 23] considered the nonlinear bending behaviour of tapered FG beams based on two different numerical approaches namely; Galerkin method and Generalized Differential Quadrature method (GDQ). Yoon et al. 24] presented a continuum based 3D beam finite element for geometrically nonlinear analysis of FG beam structures considering cross-sectional warping. Pascon 25] proposed a plane beam 
finite element to solve bending problem of FG beams assuming moderate strains and variable Poisson's ratio. In the context of geometrically exact beam theories, Eroglu [26] solved the problem of in-plane large deflections of curved FG beams with a displacement based formulation using the Variational Iteration Method (VIM) under conservative loads. Ebrahimi and Zia [27] investigated the large amplitude vibration of FG Timoshenko beams using a combined Galerkin method and multiple scales approach. Using the Ritz method, Chen et al. 28, considered the large amplitude deformations of FG porous beams by including von Kármán strain-displacement relations for free vibration and postbuckling analysis. This approach was further extended by Chen et al. 29] for the nonlinear vibration and postbuckling of FG graphene reinforced nano beams. More recently, nonlinear bending of nano porous functionally graded beams has been considered by She et al. [30, 31] for straight and curved beams, respectively. Li et al. 32] used GDQ for studying the linear and nonlinear bending of two-dimensionally FG beams. Lin et al. 33] introduced Smoothed Particle Hydrodynamics (SPM) with a Total Lagrangian (TL) formulation for the nonlinear bending analysis of FG beams with variable thickness. Using nonlocal strain gradient theory, Sahmani et al. 34] studied size effects on the nonlinear bending of micro/nano-beams reinforced with graphene. Yang et al. 35] considered the nonlinear bending of nano-beams made of bi-directional FGM using DQM. Nguyen et al. 36] investigated the ealstoplastic nonlinear bending of FG beams under various types of nonuniform distributed load.

However, except for the work of Yoon et al. 24], all of the works on the large deflection analysis of FG beams are restricted to 2D plane problems and the main focus of all of these works has been mostly on the chemical composition gradient functionally graded materials by the micro-structural mixture of metals and ceramics. Moreover, the important problem of non-conservative (follower) loads for FG beams has received little attention and as apparent from the above literature review, the 3D large deflection behaviour of functionally graded beams with porous structure has not yet been appropriately addressed in the literature.

The application of the fully intrinsic formulation of geometrically exact beams is relatively new and the potential, applicability and accuracy of this formulation are not comprehensively addressed yet in the literature. In this paper, intrinsic formulation is applied for the first time to the 3D large deflection analysis of FG porous beams under conservative and non-conservative (follower) loads. The intrinsic formulation which is free from any rotational or displacement variables, presents a number of advantages over more common displacement/rotational-based formulations including low degree of nonlinearity (quadratic at most) and bypassing the parameterisation and interpolation of finite rotations and by bringing computational efficiency and simplicity to the problem (for more details readers are referred to Khaneh Masjedi and Ovesy [37, Khaneh Masjedi and Ovesy [38] and Khaneh Masjedi and Maheri [39]). However, while the intrinsic formulation in its static form is suitable for statically determinate beams (e.g. cantilever or simply supported beams), it has difficulties in the case of statically indeterminate problems (e.g. clamped-clamped and clamped-simply supported beams) in which it is not possible to express boundary conditions without the displacement or rotational variables (Sotoudeh and Hodges [40]). In addition, based on nonlinear Green- 
Lagrange strain measures, constitutive relations are obtained for spatially variable stiffness FG porous beams.

It has been shown by Schillinger et al. 41] that computational costs are reduced with collocation methods and they can be orders of magnitude faster to achieve a certain level of accuracy compared to the finite element or Galerkin methods. Thus, we adopt the Chebyshev collocation method for the numerical discretisation and apply it to the strong form of the governing equations. The proposed collocation method contains no integration which is common to the Galerkin and finite element methods and it also shows a fast rate of convergence with fairly low order polynomials (Khaneh Masjedi and Ovesy [37). These characteristics make the Chebyshev collocation method computationally efficient in comparison with the finite element-based methods. The intrinsic formulation along with the Chebyshev collocation method presents a new paradigm in the context of the large deflection analysis of FG porous beams which is relatively efficient without any ad hoc assumption in the kinematical field description and as shown in this work it shows an excellent accuracy for the analysis of FG porous beams.

To show the robustness of the Chebyshev collocation method in the analysis of the large deflection behaviour of FGM slender structures made of porous materials, a number of classical benchmark problems are investigated. In addition to the classical benchmark problems, an example demonstrating 3D deflections of a highly flexible wing/blade made from porous material subject to combined loads representing aerodynamic loads is investigated. Various loading scenarios are considered in order to simulate different conditions and mechanisms that a typical porous structure may experience in real-life problems. The follower loading scenario imitates structures subject to engine thrust (e.g. missiles, aircraft wings), wind turbine tower and muscular forces exerted on bones, to name just a few and conservative loading scenarios corresponds to dead-weight loads. The methodology presented in this work fills the aforementioned gaps in the literature being capable of capturing 3D large deflections of FG porous beams under both conservative and nonconservative (follower) load cases. It is also noted that while the current approach is limited to a single beam, it can be further extended to more complex cases in which several beam assemblies is required by adopting multi-domain hybrid approaches such as the strong formulation finite element method [42].

The content of the current paper is outlined as follows. In Section 2 a brief description of the static intrinsic formulation of the spatially variable stiffness beams is given. In Section 3 a simplified constitutive equation is derived from the basic concepts of continuum mechanics for functionally graded beams and the effects of porosity on the elastic properties based on the semi-empirical power law are established. In Section 4 a concise description of orthogonal Chebyshev collocation and its application to the numerical discretization of the static intrinsic formulation of the beam is portrayed. In Section 5 the large deflection behaviour of FG porous beams under various load cases are studied and the obtained results based on the proposed scheme are validated against analytical results. The effects of porosity distribution for either through-the-thickness or through the beam span, are examined under both conservative and non-conservative (follower) loads. 
Finally in Section 6 some concluding remarks are drawn.

\section{Governing Equations}

\subsection{Fully Intrinsic Formulation}

The intrinsic equations of motion of a spatially variable stiffness geometrically exact beam are as follow (Khaneh Masjedi and Ovesy [37]):

$$
\begin{array}{r}
\mathbf{F}_{, 1}+\widetilde{\mathbf{K}} \cdot \mathbf{F}+\overline{\mathbf{f}}=0 \\
\mathbf{M}_{, 1}+\widetilde{\mathbf{K}} \cdot \mathbf{M}+\widetilde{\mathbf{R}}_{0,1} \cdot \mathbf{F}+\overline{\mathbf{m}}=0
\end{array}
$$

where ()$_{, 1}$ is the derivative with respect to the beam reference line $x_{1}$, " $"$ is the cross-product operator and the vector $\mathbf{K}$ is the curvature of the beam in the deformed state of the beam. $\mathbf{F}$ and $\mathbf{M}$ are the internal forces and moments and $\overline{\mathbf{f}}$ and $\overline{\mathbf{m}}$ are the external applied forces and moments, respectively.

Introducing the linear constitutive equation:

$$
\left\{\begin{array}{l}
\gamma \\
\boldsymbol{\kappa}
\end{array}\right\}=\left[\begin{array}{cc}
R & S \\
S^{T} & T
\end{array}\right]\left\{\begin{array}{c}
\mathbf{F} \\
\mathbf{M}
\end{array}\right\}
$$

where $\boldsymbol{\gamma}$ and $\boldsymbol{\kappa}$ are the vectors of generalised strains and curvatures respectively. One can eliminate $\boldsymbol{\gamma}, \boldsymbol{\kappa}$ and then only $\mathbf{F}, \mathbf{M}$ remain as unknowns. In Eq. 2.2 R $R$ and $T$ are the matrices of cross-sectional flexibility.

Eqs. 2.1) and 2.2 in conjunction with the boundary conditions constitute a complete set of equations to be numerically solved.

The governing Eq. 2.1) can be written in component form as:

$$
\begin{aligned}
& F_{1,1}+K_{2} F_{3}-K_{3} F_{2}+\bar{f}_{1}=0 \\
& F_{2,1}+K_{3} F_{1}-K_{1} F_{3}+\bar{f}_{2}=0 \\
& F_{3,1}+K_{1} F_{2}-K_{2} F_{1}+\bar{f}_{3}=0 \\
& M_{1,1}+K_{2} M_{3}-K_{3} M_{2}+2 \gamma_{12} F_{3}-2 \gamma_{13} F_{2}+\bar{m}_{1}=0 \\
& M_{2,1}+K_{3} M_{1}-K_{1} M_{3}+2 \gamma_{13} F_{1}-\left(1+\gamma_{11}\right) F_{3}+\bar{m}_{2}=0 \\
& M_{3,1}+K_{1} M_{2}-K_{2} M_{1}+\left(1+\gamma_{11}\right) F_{2}-2 \gamma_{12} F_{1}+\bar{m}_{3}=0
\end{aligned}
$$

in which, $K_{1}$ is the twist and $K_{2}$ and $K_{3}$ are the curvatures of the beam in the deformed state.

\section{Constitutive Equations}

In this section a simplified constitutive relation is obtained based on the nonlinear strain measures for the porous functionally graded beams. 


\subsection{Strain Energy}

The nonlinear strain measures can be obtained based on Green-Lagrange strains as:

$$
E_{i j}=\frac{1}{2}\left(G_{i j}-g_{i j}\right)
$$

Herein, $E_{i j}$ are the Green-Lagrange strain tensor components in the curvilinear coordinate system and $G_{i j}$ and $g_{i j}$ are the metric tensor in the deformed and undeformed state respectively. The metric tensors are defined as (Washizu [43]):

$$
\begin{aligned}
& g_{i j}=\mathbf{g}_{i} \cdot \mathbf{g}_{j} \\
& G_{i j}=\mathbf{G}_{i} \cdot \mathbf{G}_{j}
\end{aligned}
$$

Considering the $x_{1}, x_{2}$ and $x_{3}$ as the curvilinear coordinates, the tangent base vectors on the undeformed and deformed state of the beam are defined in a curvilinear coordinate system as:

$$
\begin{array}{r}
\mathbf{g}_{i}=\frac{\partial \mathbf{r}}{\partial x_{i}} \\
\mathbf{G}_{i}=\frac{\partial \mathbf{R}}{\partial x_{i}}
\end{array}
$$

Given that $\mathbf{r}_{0, i}=\mathbf{e}_{i}$, the tangent covariant base vectors in the undeformed state (i.e. $\mathbf{g}_{i}$ ) and deformed state (i.e. $\mathbf{G}_{i}$ ) of the beam can be derived from the Eqs. A.1 and A.2 as:

$$
\begin{aligned}
& \mathbf{g}_{1}=\left(1+x_{3} k_{2}-x_{2} k_{3}\right) \mathbf{e}_{1}+\left(-x_{3} k_{1}\right) \mathbf{e}_{2}+\left(x_{2} k_{1}\right) \mathbf{e}_{3} \\
& \mathbf{g}_{2}=\mathbf{e}_{2} \\
& \mathbf{g}_{3}=\mathbf{e}_{3} \\
& \mathbf{G}_{1}=\left(1+\gamma_{11}+x_{3} K_{2}-x_{2} K_{3}\right) \mathbf{e}_{1}^{*}+\left(2 \gamma_{12}-x_{3} K_{1}\right) \mathbf{e}_{2}^{*}+\left(2 \gamma_{13}+x_{2} K_{1}\right) \mathbf{e}_{3}^{*} \\
& \mathbf{G}_{2}=\mathbf{e}_{2}^{*} \\
& \mathbf{G}_{3}=\mathbf{e}_{3}^{*}
\end{aligned}
$$

From Eqs. (3.5) and (3.6) the following expressions for the metric tensor components can be derived:

$$
\begin{aligned}
& g_{11}=\left(1+x_{3} k_{2}-x_{2} k_{3}\right)+\left(x_{3} k_{1}\right)^{2}+\left(x_{2} k_{1}\right)^{2} \\
& g_{12}=g_{21}=-x_{3} k_{1} \\
& g_{13}=g_{31}=x_{2} k_{1} \\
& g_{22}=1 \\
& g_{23}=g_{32}=0 \\
& g_{33}=1
\end{aligned}
$$




$$
\begin{aligned}
& G_{11}=\left(1+\gamma_{11}+x_{3} K_{2}-x_{2} K_{3}\right)^{2}+\left(2 \gamma_{12}-x_{3} K_{1}\right)^{2}+\left(2 \gamma_{13}+x_{2} K_{1}\right)^{2} \\
& G_{12}=G_{21}=\left(2 \gamma_{12}-x_{3} K_{1}\right)^{2} \\
& G_{13}=G_{31}=\left(2 \gamma_{13}+x_{2} K_{1}\right)^{2} \\
& G_{22}=1 \\
& G_{23}=G_{32}=0 \\
& G_{33}=1
\end{aligned}
$$

The non-zero Green-Lagrange strains in terms of 1D strain measures can be shown to be in the following form:

$$
\left\{\begin{array}{c}
E_{11} \\
2 E_{12} \\
2 E_{13}
\end{array}\right\}=\left[\begin{array}{cccccc}
\sqrt{g} & -x_{3} k_{1} & x_{2} k_{1} & \left(x_{2}^{2}+x_{3}^{2}\right) k_{1} & x_{3} \sqrt{g} & -x_{2} \sqrt{g} \\
0 & 1 & 0 & -x_{3} & 0 & 0 \\
0 & 0 & 1 & x_{2} & 0 & 0
\end{array}\right]\left\{\begin{array}{c}
\gamma_{11} \\
2 \gamma_{12} \\
2 \gamma_{13} \\
\kappa_{1} \\
\kappa_{2} \\
\kappa_{3}
\end{array}\right\}
$$

where $g$ is the determinant of the metric tensor i.e. $g=\left|g_{i j}\right|$ and we have:

$$
\sqrt{g}=1-x_{2} k_{3}+x_{3} k_{2}
$$

Assuming the validity of the Hooke's law i.e. $\mathbf{S}=\mathbf{C . E}$, where $\mathbf{S}$ is denoted as the corresponding work conjugate stress measures to the Green-Lagrange strain measures, namely the 2nd Piola-Kirchhoff stresses and $\mathbf{C}$ is the constitutive tensor, the strain energy for the unit length of the beam is presented as follows:

$$
\mathcal{U}=\frac{1}{2} \int_{A}(\mathbf{E . C . E}) \sqrt{g} d A
$$

For the special case of an non-shearable straight beam (i.e. $\gamma_{12}=\gamma_{13}=0$ and $k_{1}=k_{2}=k_{3}=0$ ) with rectangular cross-section made from a functionally graded porous material, the strain energy per unit length of the beam with the help of Eq. 3.9 is written as:

$$
\mathcal{U}=\frac{1}{2}\left\{\begin{array}{c}
\gamma_{11} \\
\kappa_{1} \\
\kappa_{2} \\
\kappa_{3}
\end{array}\right\}\left[\begin{array}{cccc}
\overline{\mathcal{S}}_{11} & 0 & \overline{\mathcal{S}}_{13} & \overline{\mathcal{S}}_{14} \\
0 & \overline{\mathcal{S}}_{22} & 0 & 0 \\
\overline{\mathcal{S}}_{13} & 0 & \overline{\mathcal{S}}_{33} & \overline{\mathcal{S}}_{34} \\
\overline{\mathcal{S}}_{14} & 0 & \overline{\mathcal{S}}_{34} & \overline{\mathcal{S}}_{44}
\end{array}\right]\left\{\begin{array}{c}
\gamma_{11} \\
\kappa_{1} \\
\kappa_{2} \\
\kappa_{3}
\end{array}\right\}
$$


where:

$$
\begin{aligned}
& \overline{\mathcal{S}}_{11}=\int_{A} E\left(x_{1}, x_{2}, x_{3}\right) d A \\
& \overline{\mathcal{S}}_{22}=\int_{A} G\left(x_{1}, x_{2}, x_{3}\right)\left(x_{2}^{2}+x_{3}^{2}\right) d A \\
& \overline{\mathcal{S}}_{33}=\int_{A} E\left(x_{1}, x_{2}, x_{3}\right) x_{3}^{2} d A \\
& \overline{\mathcal{S}}_{44}=\int_{A} E\left(x_{1}, x_{2}, x_{3}\right) x_{2}^{2} d A \\
& \overline{\mathcal{S}}_{13}=\int_{A} E\left(x_{1}, x_{2}, x_{3}\right) x_{3} d A \\
& \overline{\mathcal{S}}_{14}=-\int_{A} E\left(x_{1}, x_{2}, x_{3}\right) x_{2} d A \\
& \overline{\mathcal{S}}_{34}=-\int_{A} E\left(x_{1}, x_{2}, x_{3}\right) x_{2} x_{3} d A
\end{aligned}
$$

Considering that $F_{1}=\frac{\partial \mathcal{U}}{\partial \gamma_{11}}, M_{1}=\frac{\partial \mathcal{U}}{\partial \kappa_{1}}, M_{2}=\frac{\partial \mathcal{U}}{\partial \kappa_{2}}$ and $M_{3}=\frac{\partial \mathcal{U}}{\partial \kappa_{3}}$, the simplified constitutive equations are presented as:

$$
\left\{\begin{array}{l}
\gamma_{11} \\
\kappa_{1} \\
\kappa_{2} \\
\kappa_{3}
\end{array}\right\}=\left[\begin{array}{cccc}
\overline{\mathcal{S}}_{11} & 0 & \overline{\mathcal{S}}_{13} & \overline{\mathcal{S}}_{14} \\
0 & \overline{\mathcal{S}}_{22} & 0 & 0 \\
\overline{\mathcal{S}}_{13} & 0 & \overline{\mathcal{S}}_{33} & \overline{\mathcal{S}}_{34} \\
\overline{\mathcal{S}}_{14} & 0 & \overline{\mathcal{S}}_{34} & \overline{\mathcal{S}}_{44}
\end{array}\right]^{-1}\left\{\begin{array}{c}
F_{1} \\
M_{1} \\
M_{2} \\
M_{3}
\end{array}\right\}
$$

It is noted that in order to retain the above constitutive equation in the numerical implementation the shear stiffness terms are assumed to be very large (i.e. tends to $\infty$ ).

\subsection{Porosity Distribution}

Two types of porosity distribution may be considered for the beam problem, namely; through the thickness variation and also spanwise variation which are shown in Figs. (1) and (2), respectively. The relationship between the elastic properties and porosity is recognized and semi-empirical equations are derived by curve fitting experimental data. They can be expressed in a power law form as (Kováčik [44):

$$
Y=Y_{0}\left(1-\frac{P}{P_{c}}\right)^{N_{Y}}
$$

where $Y$ is the effective elastic property of the porous material, $Y_{0}$ is the associated property of the solid material, $P$ is the porosity, $P_{c}$ is the porosity at which the effective material property is zero and $N_{Y}$ is a characteristic exponent. Subsequently one can express the Young's modulus of a porous material as:

$$
E=E_{s}\left(1-\frac{P}{P_{c}}\right)^{N_{E}}
$$


where $E$ is the effective Young's modulus of porous material with porosity $P, E_{s}$ is the Young's modulus of the solid metal, $P_{c}$ is the porosity at which the effective Young's modulus becomes zero and $N_{E}$ is the material constant often extracted from experimental data fitting. The porosity for a porous material is defined as:

$$
P=1-\frac{\rho}{\rho_{s}}
$$

where $\rho_{s}$ and $\rho_{m}$ are the density of the solid and porous material, respectively. Fitting experimental data to Eq. 3.16 often gives $P_{c}=1$. Thus considering Eqs. (3.16) and (3.17), the effective Young's modulus can be written as (Gibson and Ashby [45]):

$$
E=E_{s}\left(\frac{\rho}{\rho_{s}}\right)^{N_{E}}
$$

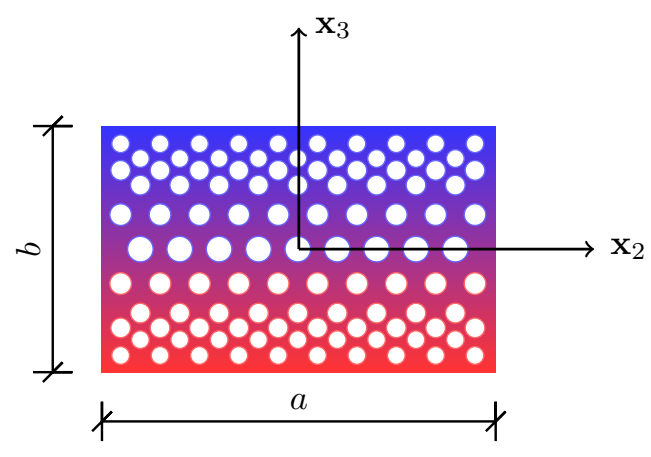

Figure 1: Porosity Distribution Through a Rectangular Beam Cross-Section Thickness

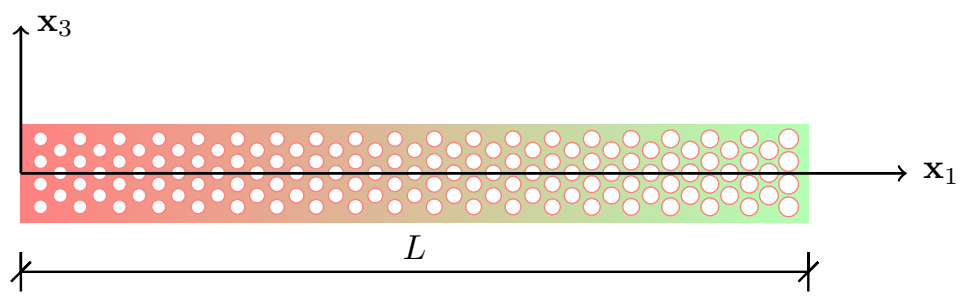

Figure 2: Spanwise Porosity Distribution

\section{Numerical Solution Scheme}

For the purpose of numerical solution of the governing equations of the beam, the Chebyshev collocation method is adopted. The Chebyshev polynomials of the first kind are employed as the trial functions for the spatial discretization of the unknown variables and the Chebyshev points are considered as collocation 
points. In the subsequent sections a brief description of the fundamentals of Chebyshev polynomials and Chebyshev collocation points is given. Please note that the content of this section is given in more detail in Khaneh Masjedi and Ovesy [37. The Chebyshev polynomials described in AppendixB section are adopted as the trial functions and the Chebyshev points are employed as the collocation points at which the residuals are minimized. The Chebyshev points are the roots of the Chebyshev polynomials of the first kind and in the interval $-1 \leqslant x \leqslant+1$ are:

$$
x^{i}=\cos \left(\frac{2 i-1}{2 N} \pi\right), \quad i=1,2, \ldots, N
$$

in which $N$ is the highest degree of the Chebyshev polynomials used as the trial functions. For an arbitrary interval $a \leqslant x \leqslant b$ the transformed Chebyshev points are:

$$
x^{i}=\frac{1}{2}(a+b)-\frac{1}{2}(b-a) \cos \left(\frac{2 i-1}{2 N} \pi\right), \quad i=1,2, \ldots, N
$$

Using the Chebyshev polynomials the variables $F$ and $M$ are discretized as:

$$
\begin{gathered}
F=\left[\begin{array}{l}
F_{1} \\
F_{2} \\
F_{3}
\end{array}\right]=\left[\begin{array}{llll}
\frac{1}{2} a_{10} & a_{11} & \ldots & a_{1 N} \\
\frac{1}{2} a_{20} & a_{21} & \ldots & a_{2 N} \\
\frac{1}{2} a_{30} & a_{31} & \ldots & a_{3 N}
\end{array}\right]\left[\begin{array}{c}
T_{0}\left(x_{1}\right) \\
T_{1}\left(x_{1}\right) \\
\vdots \\
T_{N}\left(x_{1}\right)
\end{array}\right] \\
M=\left[\begin{array}{l}
M_{1} \\
M_{2} \\
M_{3}
\end{array}\right]=\left[\begin{array}{llll}
\frac{1}{2} b_{10} & b_{11} & \ldots & b_{1 N} \\
\frac{1}{2} b_{20} & b_{21} & \ldots & b_{2 N} \\
\frac{1}{2} b_{30} & b_{31} & \ldots & b_{3 N}
\end{array}\right]\left[\begin{array}{c}
T_{0}\left(x_{1}\right) \\
T_{1}\left(x_{1}\right) \\
\vdots \\
T_{N}\left(x_{1}\right)
\end{array}\right]
\end{gathered}
$$

The total 6 boundary conditions of a cantilever beam (which is the main focus of the current paper) at the free end of the beam are denoted as:

$$
\left.\mathbf{F}\right|_{\text {at free end }}=\mathbf{F}^{t i p},\left.\quad \mathbf{M}\right|_{\text {at free end }}=\mathbf{M}^{t i p}
$$

where $\mathbf{F}^{t i p}$ and $\mathbf{M}^{t i p}$ are the vectors of applied concentrated tip forces and moments respectively. Introducing Eq.4.3 into Eq. 2.3 and setting the residuals at the Chebyshev points equal to zero in conjunction with the boundary condition equations 4.4 , constitute $6 \times(N+1)$ equations. This system of nonlinear equations will be solved for unknown coefficients $a_{i j}$ and $b_{i j}$ where $i=1,2,3$ and $j=0,1, \ldots, N$ using a Newton-Raphson scheme. 


\subsection{Post-Processing}

In order to retrieve the displacement and rotational variables from the intrinsic formulation a set of equations which relate the strains and curvatures, and displacements and rotations are presented as follow(Khaneh Masjedi and Ovesy [37]):

$$
\begin{array}{r}
\bar{\Lambda}_{, 1}=-(\widetilde{\kappa}) \bar{\Lambda} \\
R_{0,1}=\bar{\Lambda}^{T}\left(\gamma+e_{1}\right)
\end{array}
$$

where $\bar{\Lambda}$ is the matrix of linear transformation from inertial reference frame to deformed frame, $R_{0}=$ $\left[R_{1}, R_{2}, R_{3}\right]^{T}$ and $e_{1}=[1,0,0]^{T}$. By integration of Eq.4.5 the desired displacements can be obtained.

\section{Case Studies}

In this section a number of test cases are presented and the large deflection behaviour is studied under conservative and nonconservative (follower) load cases and the obtained results are validated against analytical results where available. An open cell metal foam is assumed as being representative of a porous structure and the porosity is considered to be either distributed through the beam cross-section thickness or axially along the beam span. In this work an open cell foam is considered for which $N_{E}=2, \nu=0.33$

and the shear modulus for the solid metal is denoted as; $G_{s}=\frac{E_{s}}{2(1+\nu)}$. The following characteristics are assumed for all numerical examples:

$L=1 \mathrm{~m}, \quad a=0.05 \mathrm{~m}, \quad b=0.05 \mathrm{~m}, \quad \rho_{s}=2700 \mathrm{~kg} / \mathrm{m}^{3}, \quad \rho_{m}=500 \mathrm{~kg} / \mathrm{m}^{3}, \quad E_{s}=70 \mathrm{GPa}$. It is noted that for all of the following illustrative samples, the purpose is to study the large deflection behaviour of FG beam qualitatively and show the capabilities of the proposed method. Thus, while the beam goes under large deflections, it is assumed that neither failure in the material nor any kind of elastic instability occurs.

\subsection{FG Beam with Through the Thickness Distribution of Porosity}

A porous beam made from a metal foam with rectangular cross-section is considered. The porosity is distributed through the thickness of the beam cross-section and the effective density $\rho$ is functionally graded in the $x_{3}$ direction by the power law as follows:

$$
\rho\left(x_{3}\right)=\rho_{m}+\left(\rho_{s}-\rho_{m}\right)\left(\frac{2\left|x_{3}\right|}{b}\right)^{\beta}
$$

where $\rho_{s}$ is the density of the solid metal, $\rho_{m}$ is the lowest density of the foam and $\beta$ is an exponent determining how the density or in this case porosity is distributed through the thickness. According to Eqs.(3.18) and (5.1) the density and Young's modulus distribution through a rectangular cross-section is depicted for a number of porosity levels $\beta$ in Figs.(3) and (4), respectively. 


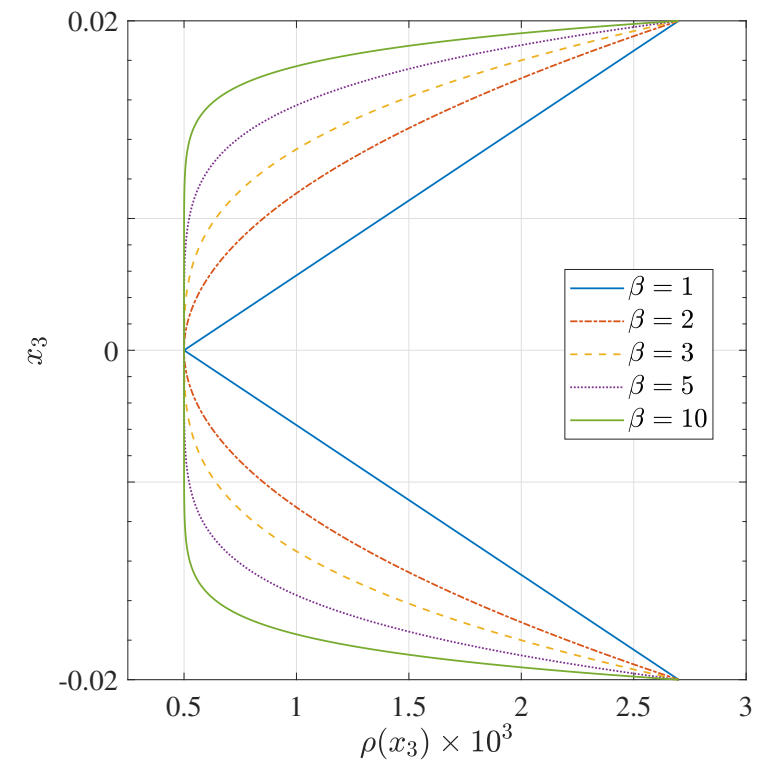

Figure 3: Density Distribution Through Beam Cross-section Thickness for Various Values of $\beta$

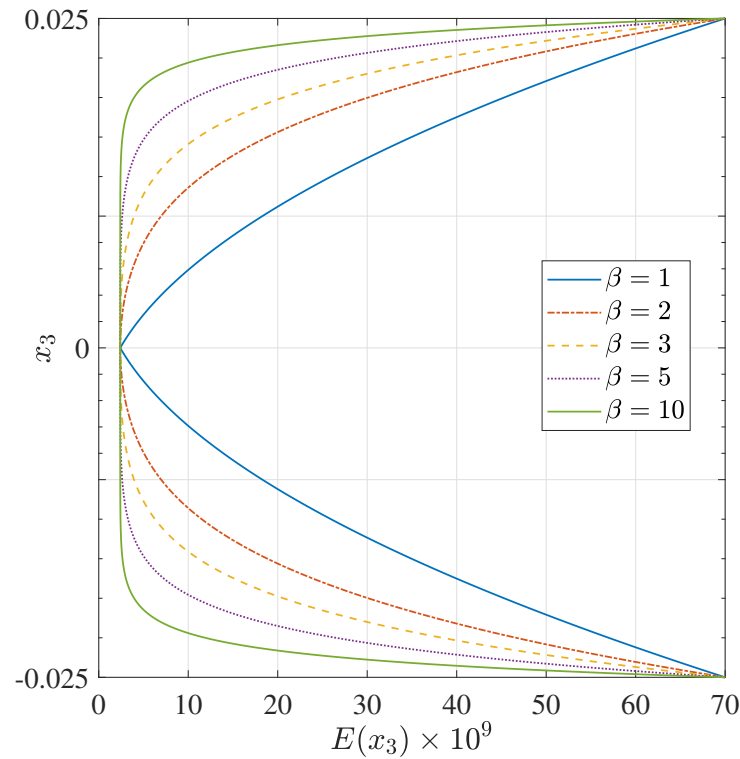

Figure 4: Young's Modulus Distribution Through Beam Cross-section Thickness for Various Values of $\beta$

\subsection{Cantilever Subject to Tip Shear Force: Linear vs Nonlinear}

In order to show the importance of including the nonlinearities in the deflection analysis of FG porous beams, a cantilever beam subject to a tip shear force is considered and the numerical results are obtained 
based on both linear and nonlinear assumptions. Additionally, the obtained results which are based on Euler-Bernoulli theory are compared to those of [46] which are based on a linear Timoshenko theory. A convergence study is performed based on the $L_{2}$ norm of the forces and moments at the clamped end. As shown in Table (1), a relatively fast convergence is obtained by using only Chebyshev polynomials of the highest degree of 8 . Table (2) depicts the tip displacement of cantilever beams for various levels of porosity $\beta$. It is noted that the exact results are obtained based on the exact solution of the linearised intrinsic governing equations for a cantilever subject to tip shear forces and moments, given by Hodges [47:

$$
U=\left[x_{1} R+\left(x_{1} L-\frac{1}{2} x_{1}^{2}\right) S \widetilde{e}_{1}-\frac{1}{2} x_{1}^{2} \widetilde{e}_{1} S^{T}-\left(\frac{1}{2} x_{1}^{2} L-\frac{1}{6} x_{1}^{3}\right) \widetilde{e}_{1} T \widetilde{e}_{1}\right] F^{t i p}+\left[x_{1} S-\frac{1}{2} x_{1}^{2} \widetilde{e}_{1} T\right] M^{t i p},
$$

where $U=\left[\begin{array}{lll}u_{1} & u_{2} & u_{3}\end{array}\right]^{T}$.

A good agreement is observed between all linear sets of results. As expected, the tip deflections predicted based on linear Timoshenko theory are larger compared to the linear Euler-Bernoulli theory. However, since the cantilever beam is relatively long and slender, this difference is negligible. On the other hand, the tip deflections obtained based on the nonlinear theory are smaller compared to both linear Euler-Bernoulli and Timoshenko theories. This behaviour occurs due to the stiffening effects of the geometric couplings in the nonlinear theory. As shown in Table (2), the difference between the tip deflections predicted by the nonlinear and linear theories increases as the porosity level $\beta$ increases.

Table 1: $L_{2}$ norm convergence

\begin{tabular}{lccccc}
\hline & \multicolumn{5}{c}{$N$ (highest degree of polynomials) } \\
\cline { 2 - 6 } & 2 & 4 & 6 & 8 & 10 \\
\hline$\|F\|_{2}$ & 4718.662 & 4716.866 & 4716.766 & 4716.766 & 4716.766 \\
$\|M\|_{2}$ & 4676.898 & 4662.740 & 4662.768 & 4662.769 & 4662.769 \\
\hline
\end{tabular}


Table 2: Tip Displacement $(\mathrm{mm})$ of FG Porous Cantilever Beam For Various Porosity Distribution

\begin{tabular}{lccccccc}
\hline & \multicolumn{5}{c}{ Euler-Bernoulli (Present) } & & \multicolumn{2}{c}{ Timoshenko [46] } \\
\cline { 2 - 4 } \cline { 7 - 8 }$\beta$ & Exact & Linear & Nonlinear & Difference* (\%) & & Exact & Linear \\
\hline 1 & 69.371 & 69.285 & 68.884 & 0.579 & & 69.600 & 69.536 \\
2 & 91.446 & 91.250 & 90.341 & 1.006 & & 91.803 & 91.675 \\
3 & 112.460 & 112.095 & 110.428 & 1.510 & & 112.935 & 112.750 \\
4 & 132.621 & 132.022 & 129.333 & 2.079 & & 133.206 & 132.970 \\
5 & 152.039 & 151.137 & 147.156 & 2.705 & & 152.726 & 152.446 \\
6 & 170.784 & 169.506 & 163.970 & 3.376 & & 171.567 & 171.248 \\
7 & 188.909 & 187.180 & 179.836 & 4.084 & & 189.779 & 189.428 \\
8 & 206.452 & 204.198 & 194.809 & 4.820 & & 207.405 & 207.024 \\
9 & 223.448 & 220.592 & 208.943 & 5.575 & & 224.477 & 224.071 \\
10 & 239.926 & 236.393 & 222.286 & 6.346 & & 241.027 & 240.599 \\
\hline
\end{tabular}

$*$ Difference $=\left|\frac{\text { Nonlinear }- \text { Linear }}{\text { Nonlinear }}\right| \times 100$

\subsubsection{Roll-Up of a Cantilever Subject to an End Moment}

In order to show the geometrical exactness of the current approach the roll-up problem due to an end moment is considered. Since there are no couplings between in-plane and out-of-plane bending deflections this problem has an analytical solution. In pure bending of a beam the reference line deforms into a part of a circle of radius $r$, therefore the reference line of the deformed beam can be defined by the angle $\theta$ and one can write:

$$
\theta=\int_{0}^{L} d \theta=\int_{0}^{L} \frac{M_{2}^{t i p}}{\overline{\mathcal{S}}_{33}} d x_{1}=\frac{M_{2}^{t i p} L}{\overline{\mathcal{S}}_{33}}
$$

Subsequently the tip deflection of the cantilever subject to an end moment is:

$$
\begin{aligned}
& u_{1}=L-r \sin \theta \\
& u_{3}=r-r \cos \theta
\end{aligned}
$$


Introducing; $r=\frac{L}{\theta}$ into Eq. 5.4:

$$
\begin{aligned}
& u_{1}=L\left(1-\frac{\sin \theta}{\theta}\right) \\
& u_{3}=L\left(\frac{1-\cos \theta}{\theta}\right)
\end{aligned}
$$

Eq. (5.3) in conjunction with Eq. (5.5) are used to obtain the analytical solution of the problem. Table (3) shows the analytical and numerical results for the end position of the FG porous beam under end moment for various load level $n=\frac{M_{2}^{t i p} L}{2 \pi \overline{\mathcal{S}}_{33}}$. Excellent agreement is observed between analytical and numerical results. Two sets of results are identical except for one case with a difference of only $0.0005 \%$. Table (4) shows the effects of porosity level on the tip deflection of FG beam under end moment $M_{R e f}=M_{2}^{t i p}=43646.6 \mathrm{~N}$.m corresponding to a moment under which the FG beam with porosity level $\beta=10$ deforms into a complete circle. The deformed configuration is depicted in Fig.(5) for various porosity distribution. As expected by increasing the porosity level $\beta$ results in more flexibility and an increase in the beam tip deflection.

Table 3: Tip Displacement of FG Porous Cantilever Beam under Tip Moment $\left(n=\frac{M_{2}^{t i p} L}{2 \pi \overline{\mathcal{S}}_{33}}\right)$

\begin{tabular}{lcclcc}
\hline & \multicolumn{2}{c}{$-u_{1} / L$} & & \multicolumn{2}{c}{$u_{3} / L$} \\
\cline { 2 - 3 } \cline { 5 - 6 }$n$ & Exact & Present & & Exact & Present \\
\hline 0.25 & 0.363380 & 0.363380 & & 0.636620 & 0.636620 \\
0.50 & 1.000000 & 1.000000 & & 0.636620 & 0.636620 \\
0.75 & 1.212207 & 1.212207 & & 0.212207 & 0.212208 \\
1.00 & 1.000000 & 1.000000 & & 0.000000 & 0.000000 \\
\hline
\end{tabular}


Table 4: Tip Displacement of FG Porous Cantilever Beam under Tip Moment $\left(M_{\text {Ref }}\right)$ for Various Porosity Distribution

\begin{tabular}{lllllll}
\hline & \multicolumn{2}{c}{$-u_{1} / L$} & & \multicolumn{2}{c}{$u_{3} / L$} \\
\cline { 2 - 3 } \cline { 6 - 6 }$\beta$ & Exact & Present & & Exact & Present \\
\hline 1 & 0.4661 & 0.4661 & & 0.6844 & 0.6844 \\
2 & 0.7163 & 0.7163 & & 0.7240 & 0.7240 \\
3 & 0.9337 & 0.9337 & & 0.6726 & 0.6726 \\
4 & 1.0937 & 1.0937 & & 0.5602 & 0.5602 \\
5 & 1.1870 & 1.1870 & & 0.4188 & 0.4188 \\
6 & 1.2172 & 1.2172 & & 0.2767 & 0.2767 \\
7 & 1.1966 & 1.1966 & & 0.1551 & 0.1551 \\
8 & 1.1422 & 1.1422 & & 0.0666 & 0.0666 \\
9 & 1.0715 & 1.0715 & & 0.0157 & 0.0157 \\
10 & 1.0000 & 1.0000 & & 0.0000 & 0.0000 \\
\hline
\end{tabular}

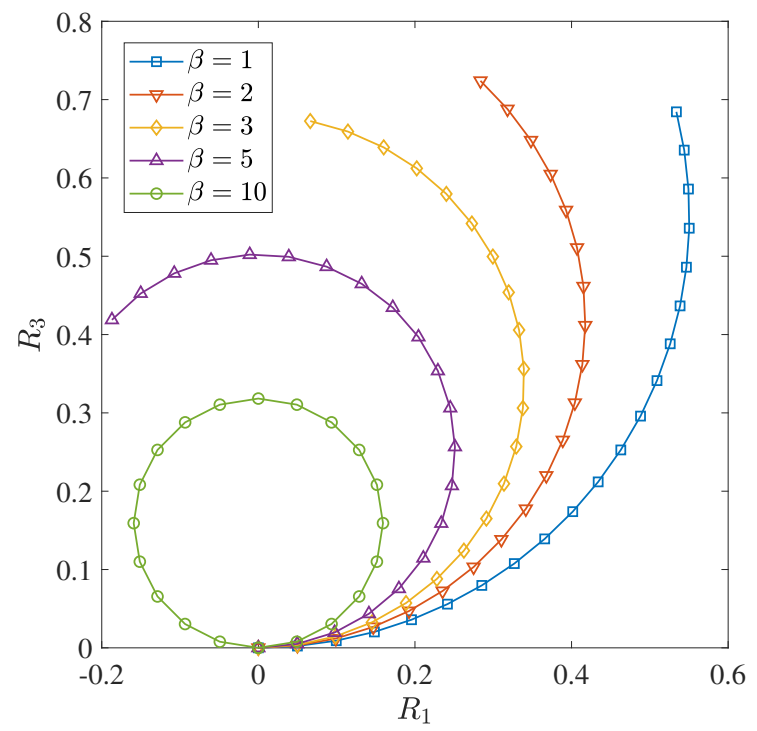

Figure 5: Deformed Configuration of FG Porous Cantilever Beam under Tip Moment $\left(M_{\text {Ref }}\right)$ for Various Porosity Distribution 


\subsubsection{Cantilever Subject to Non-Conservative (Follower) Transverse Load}

A cantilevered FG beam under non-conservative (follower) transverse tip load is considered. The orientation of the load is varying with respect to an inertial frame but it is fixed with respect to the moving frame. An analytical solution is presented by Rao and Rao 48] for the large deflection of beams under either conservative or non-conservative (follower) loads. In Table (5) the obtained numerical results are compared against analytical results of Rao and Rao [4]. Two sets of results match exactly for almost all of the load cases $\lambda=\frac{F_{3}^{t i p} L^{2}}{\overline{\mathcal{S}}_{33}}$ except for the cases of " $-R_{3} / L "$ in $\lambda=5,6,7$ where a slight difference is observed. The effects of porosity level $\beta$ on the tip deflection of the FG beam under transverse tip force $F_{3}^{t i p}=60 k N$ is given in Table (6) and the associated deformed configuration is depicted in Fig. (6) for a number of porosity level $\beta$.

Table 5: Tip Deflection of FG Porous Cantilever Beam under Non-Conservative (Follower) Tip Load For Various Load Level $\left(\lambda=\frac{F_{3}^{t i p} L^{2}}{\overline{\mathcal{S}}_{33}}\right)$

\begin{tabular}{cccccccc} 
& \multicolumn{2}{c}{$R_{1} / L$} & & \multicolumn{2}{c}{$-R_{3} / L$} & \\
\cline { 2 - 3 }$\lambda$ & Exact & Present & & Difference* $(\%)$ & Exact & Present & Difference* $(\%)$ \\
\hline 1 & 0.9356 & 0.9356 & 0.0000 & 0.3206 & 0.3206 & 0.0000 \\
2 & 0.7672 & 0.7672 & 0.0000 & 0.5737 & 0.5737 & 0.0000 \\
3 & 0.5514 & 0.5514 & 0.0000 & 0.7263 & 0.7263 & 0.0000 \\
4 & 0.3426 & 0.3426 & 0.0000 & 0.7856 & 0.7856 & 0.0000 \\
5 & 0.1722 & 0.1722 & 0.0000 & 0.7800 & 0.7799 & 0.0128 \\
6 & 0.0495 & 0.0495 & 0.0000 & 0.7395 & 0.7394 & 0.0135 \\
7 & -0.0296 & -0.0296 & 0.0000 & 0.6861 & 0.6860 & 0.0146 \\
8 & -0.0739 & -0.0739 & 0.0000 & 0.6326 & 0.6326 & 0.0000 \\
9 & -0.0924 & -0.0924 & 0.0000 & 0.5852 & 0.5852 & 0.0000 \\
10 & -0.0923 & -0.0923 & 0.0000 & 0.5458 & 0.5458 & 0.0000 \\
\hline
\end{tabular}

${ }^{*}$ Difference $=\left|\frac{\text { Exact }- \text { Present }}{\text { Exact }}\right| \times 100$ 
Table 6: Tip Displacement of FG Porous Cantilever Beam under Non-Conservative (Follower) Tip Load For various Porosity Distribution $\left(F_{3}^{t i p}=60 k N\right)$

\begin{tabular}{ccc}
\hline$\beta$ & $-u_{1} / L$ & $-u_{3} / L$ \\
\hline 1 & 0.3379 & 0.6625 \\
2 & 0.5121 & 0.7522 \\
3 & 0.6667 & 0.7866 \\
4 & 0.7935 & 0.7852 \\
5 & 0.8918 & 0.7635 \\
6 & 0.9648 & 0.7319 \\
7 & 1.0169 & 0.6970 \\
8 & 1.0525 & 0.6625 \\
9 & 1.0752 & 0.6304 \\
10 & 1.0882 & 0.6015 \\
\hline
\end{tabular}

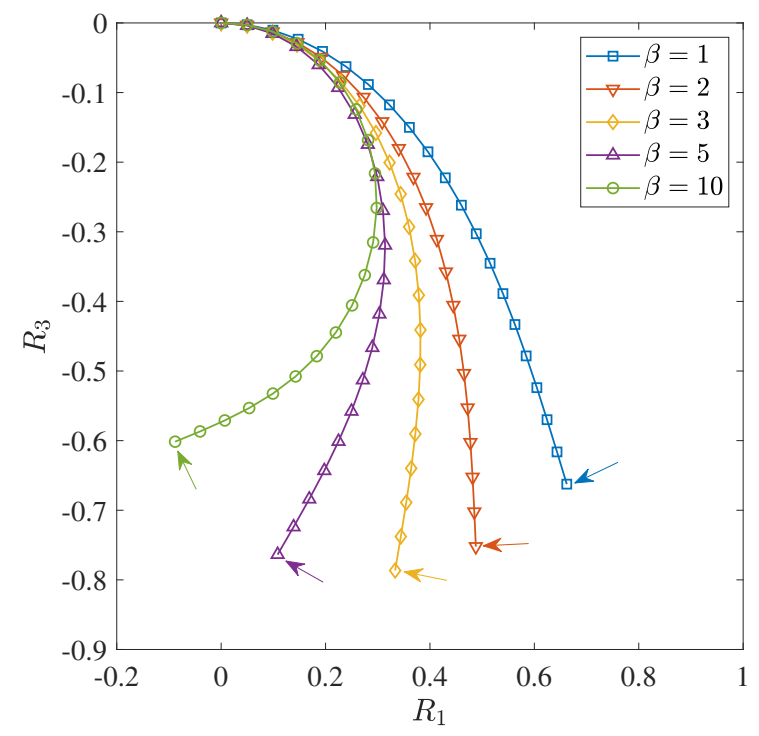

Figure 6: Tip Deflection of FG Porous Cantilever Beam under Non-Conservative (Follower) Tip Load $\left(F_{3}^{t i p}=60 k N\right)$ For Various Porosity Distribution 


\subsubsection{Cantilever Subject to Conservative Transverse Load}

A cantilevered FG beam under conservative transverse tip load is considered. In the conservative load case the orientation of the load with respect to the inertial frame is fixed in space. The numerical results are compared with the analytical results of Rao and Rao [48] in Table 77 for various load level $\lambda=\frac{P L^{2}}{\overline{\mathcal{S}}_{33}}$. A slight increase in the difference between two sets of results are observed when the load level $\lambda$ increases. However the agreement between two sets for all load levels remains excellent. The effects of porosity level on the tip deflection of FG beam under conservative transverse tip force $P=60 k N$ is given in Table (8) and the associated deformed configuration is depicted in Fig. (7) for a number of porosity levels $\beta$.

Table 7: Tip Deflection of Porous Cantilever Beam under Conservative Tip Load For Various Load Levels $\left(\lambda=\frac{P L^{2}}{\overline{\mathcal{S}}_{33}}\right)$

\begin{tabular}{|c|c|c|c|c|c|c|}
\hline \multirow[b]{2}{*}{$\lambda$} & \multicolumn{2}{|c|}{$R_{1} / L$} & \multirow[b]{2}{*}{ Difference* $(\%)$} & \multicolumn{2}{|c|}{$-R_{3} / L$} & \multirow[b]{2}{*}{ Difference* $(\%)$} \\
\hline & Exact & Present & & Exact & Present & \\
\hline 1 & 0.9436 & 0.9334 & 0.0212 & 0.3017 & 0.3026 & 0.2983 \\
\hline 2 & 0.8393 & 0.8389 & 0.0477 & 0.4935 & 0.4949 & 0.2837 \\
\hline 3 & 0.7456 & 0.7450 & 0.0805 & 0.6033 & 0.6052 & 0.3149 \\
\hline 4 & 0.6711 & 0.6704 & 0.1043 & 0.6700 & 0.6723 & 0.3433 \\
\hline 5 & 0.6124 & 0.6117 & 0.1143 & 0.7138 & 0.7166 & 0.3923 \\
\hline 6 & 0.5654 & 0.5647 & 0.1238 & 0.7446 & 0.7478 & 0.4298 \\
\hline 7 & 0.5271 & 0.5263 & 0.1518 & 0.7674 & 0.7710 & 0.4691 \\
\hline 8 & 0.4952 & 0.4944 & 0.1615 & 0.7850 & 0.7890 & 0.5095 \\
\hline 9 & 0.4682 & 0.4673 & 0.1922 & 0.7991 & 0.8035 & 0.5506 \\
\hline 10 & 0.4450 & 0.4439 & 0.2472 & 0.8106 & 0.8167 & 0.7525 \\
\hline
\end{tabular}

$*$ Difference $=\left|\frac{\text { Exact }- \text { Present }}{\text { Exact }}\right| \times 100$ 
Table 8: Tip Deflection of FG Porous Cantilever Beam under Conservative Tip Load For Various Porosity Distribution $(P=60 k N)$

\begin{tabular}{ccc}
\hline$\beta$ & $-u_{1} / L$ & $-u_{3} / L$ \\
\hline 1 & 0.2095 & 0.5558 \\
2 & 0.2778 & 0.6268 \\
3 & 0.3318 & 0.6736 \\
4 & 0.3752 & 0.7067 \\
5 & 0.4107 & 0.7313 \\
6 & 0.4403 & 0.7503 \\
7 & 0.4654 & 0.7655 \\
8 & 0.4870 & 0.7780 \\
9 & 0.5056 & 0.7883 \\
10 & 0.5220 & 0.7972 \\
\hline
\end{tabular}

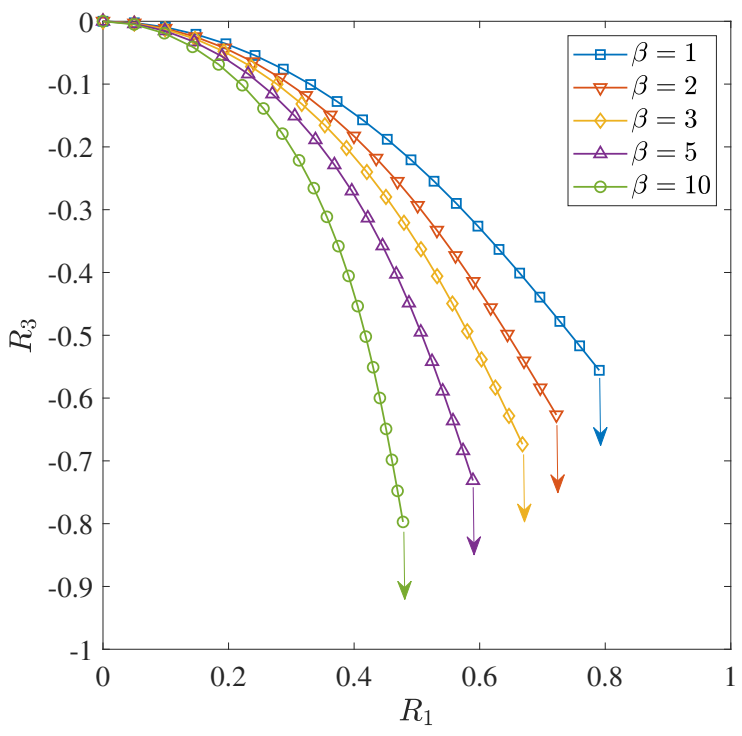

Figure 7: Deformed Configuration of FG Porous Cantilever Beam under Conservative Tip Load For Various Porosity Distribution $(P=60 k N)$ 


\subsection{FG Beam with Axial Distribution of Porosity}

A porous beam which is functionally graded axially (i.e. exhibiting spanwise variable stiffness) along its span is considered in this section. The effective density $\rho$ is functionally graded in the $x_{1}$ direction by the power law as follows:

$$
\rho\left(x_{1}\right)=\rho_{m}+\left(\rho_{s}-\rho_{m}\right)\left(1-\frac{x_{1}}{L}\right)^{\beta}
$$

According to Eqs. (3.18) and (5.6) the spanwise density and Young's modulus distribution is depicted for a number of porosity levels $\beta$ in Figs. (8) and (9), respectively.

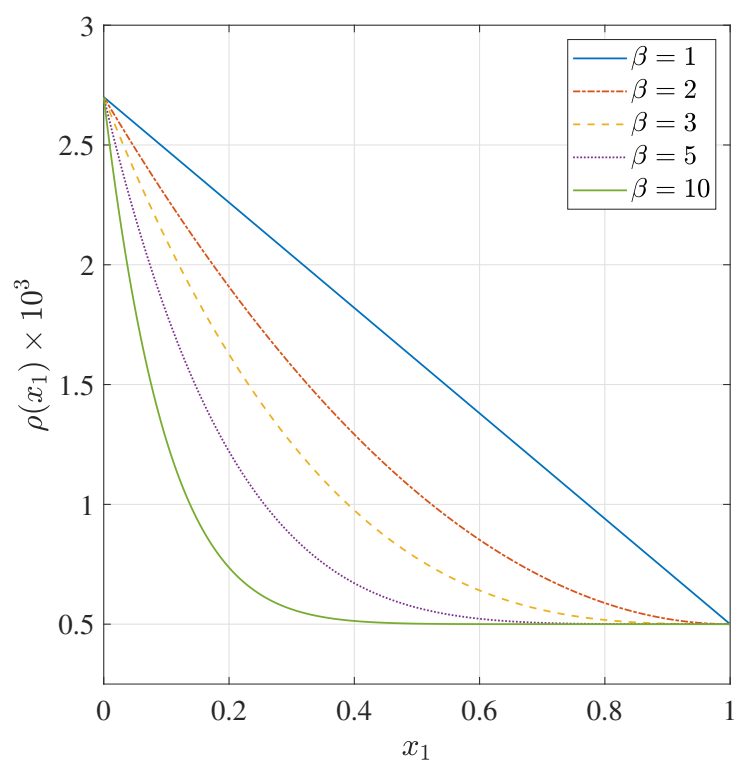

Figure 8: Density Distribution Along Beam Span for Various Values of $\beta$ 


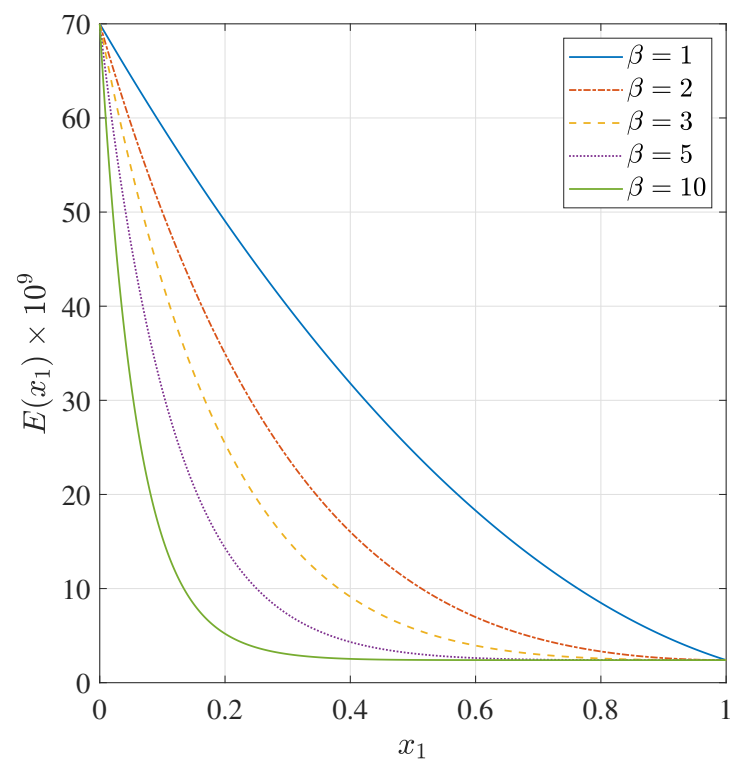

Figure 9: Young's Modulus Distribution Along Beam Span for Various Values of $\beta$

\subsubsection{Roll-Up of a Cantilever Subject to an End Moment}

The roll-up problem is considered under a tip moment. The convergence study is shown in Table (9). Using polynomials with the highest degree of 14 , results in a converged solution. A comparison of this study and those of Table (1) reveals that for obtaining converged results in the case of axially distributed porosity, higher level of discretisation is required. This can be attributed to the fact that for the FG beam with axially distributed porosity a finer descritisation including a higher number of collocation points is necessary to capture the spanwise variation of stiffness properties compared to the FG beam with through the thickness distribution of porosity.

The tip displacement of the cantilevered FG beam under various load levels are given in Table 10 for different porosity distributions. The deformed configurations are depicted in Figs. (10) under tip moment of $M_{2}^{t i p}=10 k N . m$ for a number of porosity levels $\beta$. As shown, due to the functionally distributed of elastic properties along the beam span the beam does not undergo a deflection with a uniform curvature as was the case of an FG beam with through the beam cross-section porosity distribution. The deformed state of the beam has a distribution of curvature along its span which decreases from its free end to the clamped end as expected due to the increase of Young's modulus toward the clamped end. 
Table 9: $L_{2}$ norm convergence

\begin{tabular}{ccccccccc}
\hline & \multicolumn{7}{c}{$N$ (highest degree of polynomials) } \\
\cline { 2 - 8 } & 4 & 6 & 8 & 10 & 12 & 14 & 16 \\
\hline$\|F\|_{2}$ & 7.681 & 19.229 & 20.310 & 20.351 & 20.355 & 20.356 & 20.356 \\
$\|M\|_{2}$ & 10000.201 & 10001.884 & 10002.063 & 10002.070 & 10002.072 & 10002.072 & 10002.072 \\
\hline
\end{tabular}

Table 10: Tip Displacement of FG Porous Cantilever Beam For Various Tip Moments and Porosity Distribution

\begin{tabular}{|c|c|c|c|c|c|c|c|c|c|c|}
\hline \multirow[b]{2}{*}{$\beta$} & \multicolumn{2}{|c|}{$2 k N . m$} & \multicolumn{2}{|c|}{$4 k N . m$} & \multicolumn{2}{|c|}{$6 k N . m$} & \multicolumn{2}{|c|}{$8 k N . m$} & \multicolumn{2}{|c|}{$10 k N . m$} \\
\hline & $-u_{1} / L$ & $u_{3} / L$ & $-u_{1} / L$ & $u_{3} / L$ & $-u_{1} / L$ & $u_{3} / L$ & $-u_{1} / L$ & $u_{3} / L$ & $-u_{1} / L$ & $u_{3} / L$ \\
\hline 1 & 0.0051 & 0.0717 & 0.0202 & 0.1415 & 0.0447 & 0.2076 & 0.0777 & 0.2684 & 0.1179 & 0.3225 \\
\hline 2 & 0.0234 & 0.1449 & 0.0894 & 0.2717 & 0.1867 & 0.3657 & 0.2990 & 0.4192 & 0.4088 & 0.4320 \\
\hline 3 & 0.0504 & 0.2143 & 0.1849 & 0.3759 & 0.3607 & 0.4516 & 0.5255 & 0.4392 & 0.6379 & 0.3656 \\
\hline 4 & 0.0784 & 0.2710 & 0.2778 & 0.4467 & 0.5102 & 0.4804 & 0.6841 & 0.3945 & 0.7499 & 0.2619 \\
\hline 5 & 0.1042 & 0.3161 & 0.3586 & 0.4939 & 0.6269 & 0.4808 & 0.7848 & 0.3356 & 0.7943 & 0.1789 \\
\hline 6 & 0.1271 & 0.3522 & 0.4267 & 0.5260 & 0.7161 & 0.4690 & 0.8481 & 0.2809 & 0.8084 & 0.1224 \\
\hline 7 & 0.1470 & 0.3815 & 0.4838 & 0.5484 & 0.7850 & 0.4529 & 0.8887 & 0.2348 & 0.8103 & 0.0862 \\
\hline 8 & 0.1644 & 0.4057 & 0.5317 & 0.5645 & 0.8389 & 0.4358 & 0.9153 & 0.1973 & 0.8078 & 0.0637 \\
\hline 9 & 0.1795 & 0.4259 & 0.5723 & 0.5764 & 0.8818 & 0.4194 & 0.9332 & 0.1670 & 0.8043 & 0.0503 \\
\hline 10 & 0.1927 & 0.4430 & 0.6071 & 0.5853 & 0.9166 & 0.4040 & 0.9456 & 0.1425 & 0.8011 & 0.0426 \\
\hline
\end{tabular}




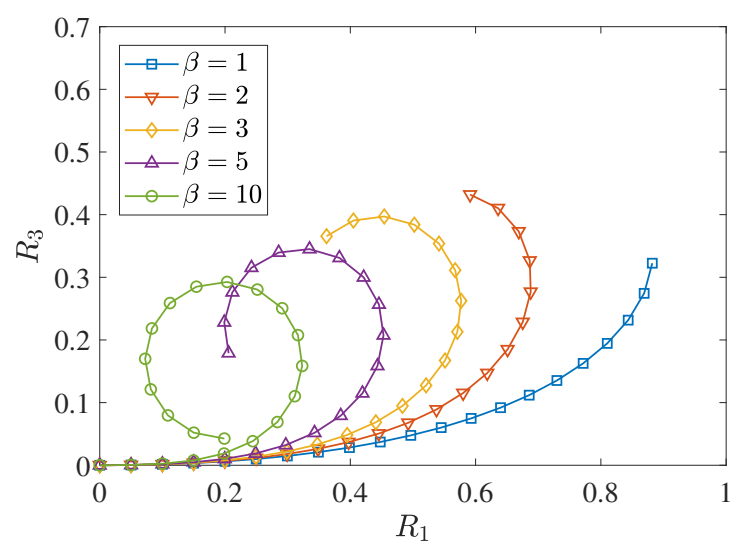

Figure 10: Deformed Configuration of Rolled-Up FG Porous Cantilever Beam with Various Porosity Distribution Under Tip Moment $\left(M_{2}^{\text {tip }}=10 k N . m\right)$

\subsubsection{Cantilever Subject to Non-Conservative (Follower) Transverse Load}

A cantilevered axially FG beam under non-conservative (follower) transverse tip load is considered. The effects of porosity distribution level $\beta$ on the tip deflection of FG beam under a number of non-conservative (follower) transverse tip forces are given in Table (11) and the deformed configurations for a number of porosity distribution levels $\beta$ under a tip transverse force of $F_{3}^{t i p}=20 k N$ are depicted in Fig. (12). It is observed that due to the lower Young's modulus near tip regions compared to the clamped end regions, the curvature of deformation increases. This behaviour becomes even more apparent when the level of porosity increases. 
Table 11: Tip Displacement of FG Porous Cantilever Beam For Various Transverse Non-Conservative (Follower) Tip Load and Porosity Distribution

\begin{tabular}{|c|c|c|c|c|c|c|c|c|c|c|}
\hline \multirow[b]{2}{*}{$\beta$} & \multicolumn{2}{|c|}{$4 k N$} & \multicolumn{2}{|c|}{$8 k N$} & \multicolumn{2}{|c|}{$12 k N$} & \multicolumn{2}{|c|}{$16 k N$} & \multicolumn{2}{|c|}{$20 k N$} \\
\hline & $-u_{1} / L$ & $u_{3} / L$ & $-u_{1} / L$ & $u_{3} / L$ & $-u_{1} / L$ & $u_{3} / L$ & $-u_{1} / L$ & $u_{3} / L$ & $-u_{1} / L$ & $u_{3} / L$ \\
\hline 1 & 0.0033 & 0.0687 & 0.0128 & 0.1346 & 0.0272 & 0.1956 & 0.0451 & 0.2507 & 0.0651 & 0.2995 \\
\hline 2 & 0.0123 & 0.1240 & 0.0427 & 0.2295 & 0.0804 & 0.3116 & 0.1182 & 0.3741 & 0.1533 & 0.4223 \\
\hline 3 & 0.0280 & 0.1840 & 0.0853 & 0.3158 & 0.1426 & 0.4013 & 0.1916 & 0.4589 & 0.2324 & 0.5003 \\
\hline 4 & 0.0464 & 0.2371 & 0.1267 & 0.3812 & 0.1956 & 0.4636 & 0.2495 & 0.5155 & 0.2919 & 0.5516 \\
\hline 5 & 0.0647 & 0.2809 & 0.1621 & 0.4302 & 0.2378 & 0.5085 & 0.2940 & 0.5559 & 0.3368 & 0.5884 \\
\hline 6 & 0.0815 & 0.3167 & 0.1916 & 0.4677 & 0.2771 & 0.5423 & 0.3287 & 0.5865 & 0.3715 & 0.6164 \\
\hline 7 & 0.0963 & 0.3461 & 0.2161 & 0.4973 & 0.2987 & 0.5689 & 0.3565 & 0.6107 & 0.3991 & 0.6388 \\
\hline 8 & 0.1094 & 0.3705 & 0.2365 & 0.5212 & 0.3211 & 0.5904 & 0.3792 & 0.6303 & 0.4216 & 0.6570 \\
\hline 9 & 0.1209 & 0.3910 & 0.2538 & 0.5410 & 0.3398 & 0.6082 & 0.3981 & 0.6466 & 0.4404 & 0.6722 \\
\hline 10 & 0.1310 & 0.4084 & 0.2686 & 0.5576 & 0.3557 & 0.6232 & 0.4141 & 0.6604 & 0.4562 & 0.6852 \\
\hline
\end{tabular}

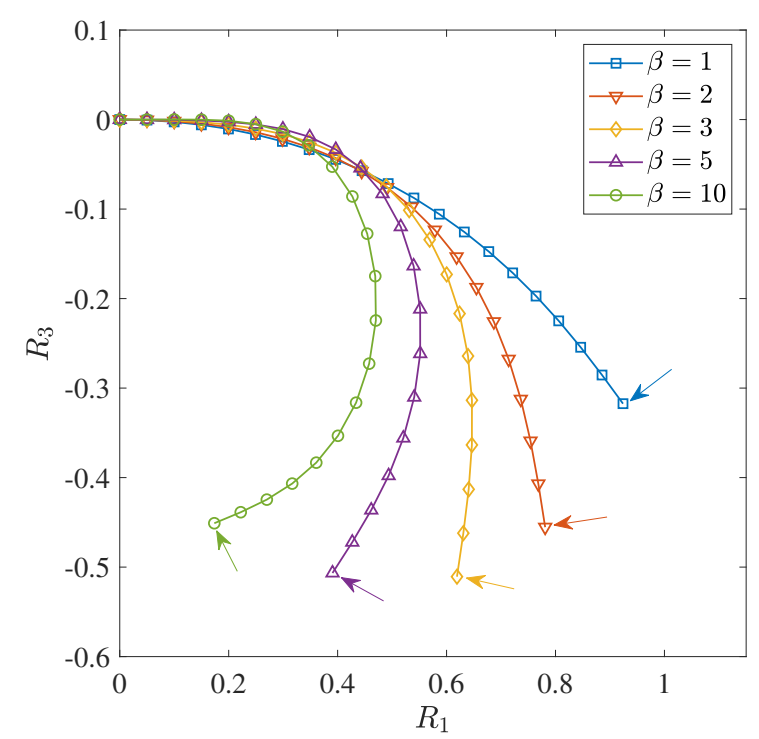

Figure 11: Deformed Configuration of FG Porous Cantilever Beam with Various Porosity Distribution Under Non-Conservative (Follower) Tip Load $\left(F_{3}^{t i p}=20 k N\right)$ 


\subsubsection{Cantilever Subject to Conservative Transverse Load}

A cantilevered axially FG beam under conservative transverse tip load is considered. Table (12) shows the effects of porosity distribution levels $\beta$ on the tip deflection of FG beam under a number of conservative transverse tip forces. The deformed configurations for a number of porosity distribution level $\beta$ under a tip transverse force of $P=20 k N$ are depicted in Fig. (12). It is observed that when the level of porosity increases the overall beam stiffness decreases and as expected beam undergoes larger deformations.

Table 12: Tip Displacement of FG Porous Cantilever Beam For Various Transverse Conservative Tip Load and Porosity Distribution

\begin{tabular}{|c|c|c|c|c|c|c|c|c|c|c|}
\hline \multirow[b]{2}{*}{$\beta$} & \multicolumn{2}{|c|}{$4 k N$} & \multicolumn{2}{|c|}{$8 k N$} & \multicolumn{2}{|c|}{$12 k N$} & \multicolumn{2}{|c|}{$16 k N$} & \multicolumn{2}{|c|}{$20 k N$} \\
\hline & $-u_{1} / L$ & $u_{3} / L$ & $-u_{1} / L$ & $u_{3} / L$ & $-u_{1} / L$ & $u_{3} / L$ & $-u_{1} / L$ & $u_{3} / L$ & $-u_{1} / L$ & $u_{3} / L$ \\
\hline 1 & 0.0034 & 0.0689 & 0.0133 & 0.1364 & 0.0292 & 0.2010 & 0.0505 & 0.2617 & 0.0761 & 0.3174 \\
\hline 2 & 0.0128 & 0.1258 & 0.0485 & 0.2404 & 0.1004 & 0.3355 & 0.1598 & 0.4071 & 0.2190 & 0.4554 \\
\hline 3 & 0.0306 & 0.1907 & 0.1100 & 0.3457 & 0.2102 & 0.4472 & 0.3054 & 0.4978 & 0.3809 & 0.5107 \\
\hline 4 & 0.0537 & 0.2516 & 0.1828 & 0.4312 & 0.3252 & 0.5187 & 0.4388 & 0.5369 & 0.5120 & 0.5171 \\
\hline 5 & 0.0788 & 0.3046 & 0.2553 & 0.4955 & 0.4280 & 0.5602 & 0.5465 & 0.5487 & 0.6094 & 0.5066 \\
\hline 6 & 0.1037 & 0.3496 & 0.3219 & 0.5429 & 0.5145 & 0.5833 & 0.6305 & 0.5482 & 0.6808 & 0.4924 \\
\hline 7 & 0.1273 & 0.3875 & 0.3812 & 0.5779 & 0.5861 & 0.5956 & 0.6958 & 0.5428 & 0.7337 & 0.4790 \\
\hline 8 & 0.1493 & 0.4196 & 0.4331 & 0.6042 & 0.6450 & 0.6017 & 0.7459 & 0.5358 & 0.7732 & 0.4676 \\
\hline 9 & 0.1694 & 0.4469 & 0.4783 & 0.6241 & 0.6938 & 0.6041 & 0.7873 & 0.5286 & 0.8033 & 0.4583 \\
\hline 10 & 0.1877 & 0.4703 & 0.5177 & 0.6395 & 0.7344 & 0.6046 & 0.8197 & 0.5220 & 0.8264 & 0.4510 \\
\hline
\end{tabular}




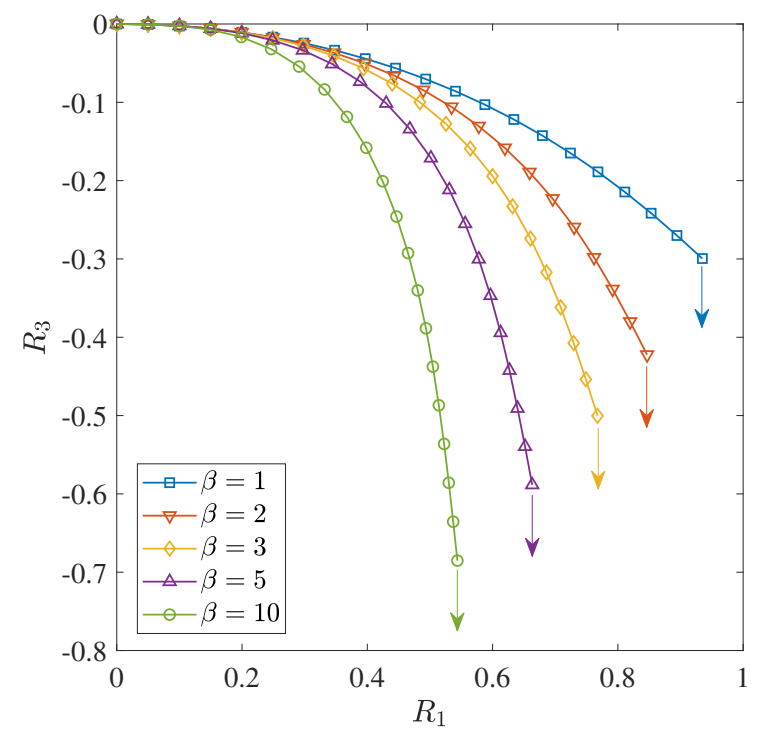

Figure 12: Deformed Configuration of FG Porous Cantilever Beam with Various Porosity Distribution Under Conservative Tip Load $(P=20 k N)$

\subsubsection{Cantilever Subject to Combined Shear Loads}

A cantilevered axially FG beam subject to combined follower tip shear loads (i.e. $F_{2}^{t i p}$ and $F_{3}^{t i p}$ ) is considered. Under the action of the combined load, the FG beam undergoes large 3D deflections. In order to verify the results obtained from the proposed method, a finite element (FE) model is developed in ABAQUS using 32 beam elements of type "B31". Young's modulus $\left(E\left(x_{1}\right)\right)$ is considered to be constant along each element and its value is assumed to be equal to the value of $E\left(x_{1}\right)$ at the middle point of each element. Table 13 shows the 3D tip displacement of FG beam subject to $F_{2}^{t i p}=2 k N$ and $F_{2}^{t i p}=3 k N$ for various porosity distributions $\beta$ obtained from the FE and present model. Very good agreement is observed between two sets of results. The deformed configuration is depicted in Fig. 13 for a number of porosity distribution $\beta$. 
Table 13: Tip Displacement of FG Porous Cantilever Beam under Combined Shear Loads $\left(F_{2}^{t i p}=2 k N, F_{2}^{t i p}=3 k N\right)$

\begin{tabular}{ccccccccc}
\hline & \multicolumn{2}{c}{$-u_{1} / L$} & & \multicolumn{2}{c}{$u_{2} / L$} & & \multicolumn{2}{c}{$u_{3} / L$} \\
\cline { 2 - 3 } \cline { 8 - 9 }$\beta$ & FE & Present & & FE & Present & & FE & Present \\
\hline 1 & 0.0027 & 0.0028 & & 0.0347 & 0.0347 & & 0.0517 & 0.0520 \\
2 & 0.0104 & 0.0105 & & 0.0631 & 0.0635 & & 0.0947 & 0.0105 \\
3 & 0.0250 & 0.0253 & & 0.0960 & 0.0965 & & 0.1440 & 0.1448 \\
4 & 0.0441 & 0.0445 & & 0.1271 & 0.1277 & & 0.1907 & 0.1916 \\
5 & 0.0650 & 0.0654 & & 0.1545 & 0.1551 & & 0.2317 & 0.2327 \\
6 & 0.0857 & 0.0863 & & 0.1779 & 0.1786 & & 0.2668 & 0.2678 \\
7 & 0.1056 & 0.1062 & & 0.1978 & 0.1985 & & 0.2967 & 0.2977 \\
8 & 0.1241 & 0.1248 & & 0.2148 & 0.2155 & & 0.3221 & 0.3232 \\
9 & 0.1411 & 0.1418 & & 0.2293 & 0.2300 & & 0.3440 & 0.3450 \\
10 & 0.1567 & 0.1574 & & 0.2418 & 0.2425 & & 0.3628 & 0.3618 \\
\hline
\end{tabular}

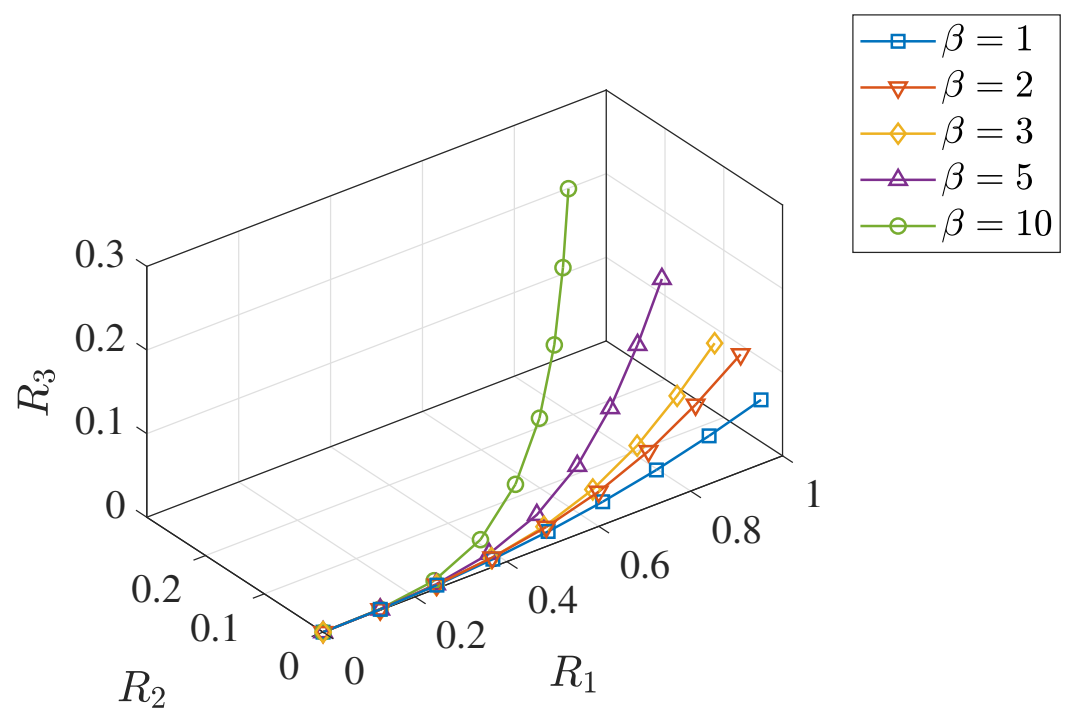

Figure 13: Deformed Configuration of FG Porous Cantilever Beam Under Combined Shear Tip Loads $\left(F_{2}^{t i p}=2 k N, F_{2}^{t i p}=\right.$ $3 k N)$ for Various Porosity Distribution 


\subsubsection{Cantilever Subject to Combined Loads}

A cantilevered axially FG beam under a combined load of tip twist moment (i.e. $M_{1}^{\text {tip }}$ ) and transverse tip force (i.e. $F_{3}^{\text {tip }}$ ) is considered in this test case. Due to the coupling of in- and out-of-plane bending, the FG beam undergoes a $3 \mathrm{D}$ deflection. The motivation for this test case is the aeroelastic response of structures such as aircraft wings and wind turbine rotor blades under the action of aerodynamic forces which are considered as nonconservative (follower) loads (Argyris and Symeonidis 49]). The 3D displacement of an axially FG beam under combined load of $M_{1}^{t i p}=1 k N . m$ and $F_{3}^{t i p}=10 k N$ is presented in Table 14 for various porosity distributions $\beta$. The deformed configuration is depicted in Fig. (14 for a number of porosity distribution $\beta$.

Table 14: Tip Displacement of FG Porous Cantilever Beam Under Combined Tip Load $\left(M_{1}^{t i p}=1 k N . m, F_{3}^{t i p}=10 k N\right)$ for Various Porosity Distribution

\begin{tabular}{ccccc}
\hline \multicolumn{1}{c}{$\beta$} & & $-u_{1} / L$ & $u_{2} / L$ & $u_{3} / L$ \\
\cline { 1 - 1 } \cline { 5 - 6 } 2 & & 0.0205 & 0.0269 & 0.1669 \\
2 & & 0.0726 & 0.0855 & 0.2772 \\
3 & & 0.1581 & 0.1534 & 0.3715 \\
4 & & 0.2532 & 0.2130 & 0.4342 \\
5 & & 0.3428 & 0.2603 & 0.4718 \\
6 & & 0.4215 & 0.2967 & 0.4931 \\
7 & & 0.4889 & 0.3248 & 0.5043 \\
8 & & 0.5460 & 0.3465 & 0.5095 \\
9 & & 0.5944 & 0.3637 & 0.5112 \\
10 & & 0.6356 & 0.3774 & 0.5107 \\
\hline
\end{tabular}




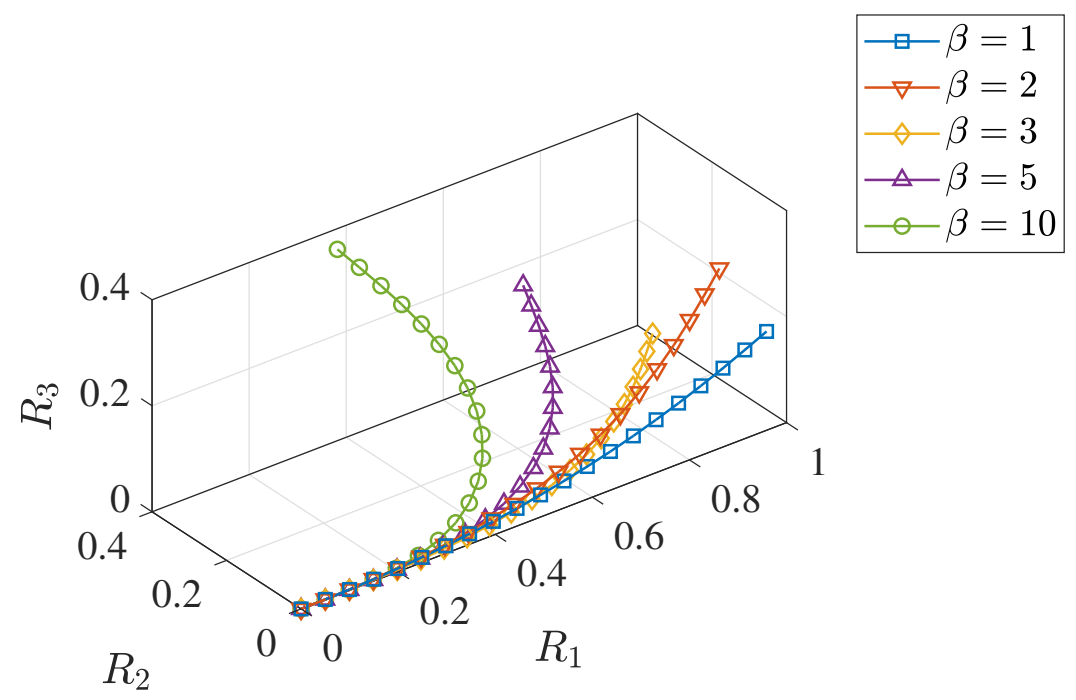

Figure 14: Deformed Configuration of FG Porous Cantilever Beam Under Combined Tip Load $\left(M_{1}^{t i p}=1 k N \cdot m, F_{3}^{t i p}=10 k N\right)$ for Various Porosity Distribution

\section{Summary and Conclusion}

Functionally graded porous materials offer promising multifunctional properties for the optimal design of structures with minimal weight, so they are favourable options for engineering design. Slender structures showing large deflections are involved in many engineering applications. For addressing this problem, a spatially variable stiffness geometrically exact beam model with fully intrinsic formulation showing a very low degree of nonlinearity is presented for the large deflection analysis of slender beam-like porous structures under conservative or non-conservative (follower) load cases. An orthogonal Chebyshev collocation method is adopted for the numerical discretization of the governing equations which is simple to implement and bypasses the integrations common in finite element and Galerkin methods. However, the proposed method is appropriate only for statically determinate problems. A number of test cases have been considered for two types of porosity distribution, namely; a distribution through the beam cross-section and an axial distribution along the beam span where results are compared to available analytical solutions. While the focus of the numerical samples has been on classical problems, it gives fundamental understanding of the large deflection mechanism of functionally graded porous structures. The proposed method can model both conservative and non-conservative (follower) load cases and while being relatively accurate, presents a versatile computationally efficient tool for the $3 \mathrm{D}$ large deflection analysis of variable stiffness functionally graded porous structures. 


\section{References}

[1] Saavedra Flores EI, Friswell MI, Xia Y. Variable stiffness biological and bio-inspired materials. Journal of Intelligent Material Systems and Structures 2013;24(5):529-40.

[2] Rafsanjani A, Brulé V, Western TL, Pasini D. Hydro-responsive curling of the resurrection plant selaginella lepidophylla. Scientific reports $2015 ; 5$.

[3] Zhao ZL, Liu ZY, Feng XQ. Chirality-dependent flutter of typha blades in wind. Scientific reports 2016;6:28907.

[4] Gonzalez OM, Nguyen KA. Senile coconut palms: Functional design and biomechanics of stem green tissue. Wood Material Science \& Engineering 2017;12(2):98-117.

[5] Bottlang M, Long WB. Biomechanics of rib fracture fixation. In: Injuries to the Chest Wall. Springer; 2015, p. 53-71.

[6] Heyland M, Trepczynski A, Duda GN, Zehn M, Schaser KD, Märdian S. Selecting boundary conditions in physiological strain analysis of the femur: Balanced loads, inertia relief method and follower load. Medical engineering \& physics 2015;37(12):1180-5.

[7] Birdwell JA, Solomon JH, Thajchayapong M, Taylor MA, Cheely M, Towal RB, et al. Biomechanical models for radial distance determination by the rat vibrissal system. Journal of Neurophysiology 2007;98(4):2439-55.

[8] Li F, Liu W, Stefanini C, Fu X, Dario P. A novel bioinspired pvdf micro/nano hair receptor for a robot sensing system. Sensors 2010;10(1):994-1011.

[9] Mongeau JM, Demir A, Dallmann CJ, Jayaram K, Cowan NJ, Full RJ. Mechanical processing via passive dynamic properties of the cockroach antenna can facilitate control during rapid running. Journal of Experimental Biology 2014;217(18):3333-45.

[10] Trivedi D, Rahn CD, Kier WM, Walker ID. Soft robotics: Biological inspiration, state of the art, and future research. Applied Bionics and Biomechanics 2008;5(3):99-117.

[11] Mac Murray BC, An X, Robinson SS, van Meerbeek IM, O'Brien KW, Zhao H, et al. Poroelastic foams for simple fabrication of complex soft robots. Advanced Materials 2015;27(41):6334-40.

[12] Parthasarathy J, Starly B, Raman S. A design for the additive manufacture of functionally graded porous structures with tailored mechanical properties for biomedical applications. Journal of Manufacturing Processes 2011;13(2):160-70.

[13] Kang YA, Li XF. Bending of functionally graded cantilever beam with power-law non-linearity subjected to an end force. International Journal of Non-Linear Mechanics 2009;44(6):696-703.

[14] Kang YA, Li XF. Large deflections of a non-linear cantilever functionally graded beam. Journal of Reinforced Plastics and Composites 2010;29(12):1761-74.

[15] Rahimi GH, Davoodinik AR. Large deflection of functionally graded cantilever flexible beam with geometric non-linearity: Analytical and numerical approaches. Scientia Iranica Transaction B, Mechanical Engineering 2010;17(1):25.

[16] Kocatürk T, Şimşek M, Akbaş SD. Large displacement static analysis of a cantilever timoshenko beam composed of functionally graded material. Science and Engineering of Composite Materials 2011;18(1-2):21-34.

[17] Almeida CA, Albino JCR, Menezes IFM, Paulino GH. Geometric nonlinear analyses of functionally graded beams using a tailored lagrangian formulation. Mechanics Research Communications 2011;38(8):553-9.

[18] Zhang DG. Nonlinear bending analysis of fgm beams based on physical neutral surface and high order shear deformation theory. Composite Structures 2013;100:121-6.

[19] Nguyen DK. Large displacement response of tapered cantilever beams made of axially functionally graded material. Composites Part B: Engineering 2013;55:298-305.

[20] Nguyen DK. Large displacement behaviour of tapered cantilever euler-bernoulli beams made of functionally graded material. Applied Mathematics and Computation 2014;237:340-55.

[21] Nguyen DK, Gan BS. Large deflections of tapered functionally graded beams subjected to end forces. Applied Mathematical Modelling 2014;38(11-12):3054-66. 
[22] Sitar M, Kosel F, Brojan M. Large deflections of nonlinearly elastic functionally graded composite beams. Archives of Civil and Mechanical Engineering 2014;14(4):700-9.

[23] Niknam H, Fallah A, Aghdam M. Nonlinear bending of functionally graded tapered beams subjected to thermal and mechanical loading. International Journal of Non-Linear Mechanics 2014;65:141-7.

[24] Yoon K, Lee PS, Kim DN. Geometrically nonlinear finite element analysis of functionally graded 3d beams considering warping effects. Composite Structures 2015;132:1231-47.

[25] Pascon JP. Finite element analysis of flexible functionally graded beams with variable poissons ratio. Engineering Computations 2016;33(8):2421-47.

[26] Eroglu U. Large deflection analysis of planar curved beams made of functionally graded materials using variational iterational method. Composite Structures 2016;136:204-16.

[27] Ebrahimi F, Zia M. Large amplitude nonlinear vibration analysis of functionally graded timoshenko beams with porosities. Acta Astronautica 2015;116:117-25.

[28] Chen D, Kitipornchai S, Yang J. Nonlinear free vibration of shear deformable sandwich beam with a functionally graded porous core. Thin-Walled Structures 2016;107:39-48.

[29] Chen D, Yang J, Kitipornchai S. Nonlinear vibration and postbuckling of functionally graded graphene reinforced porous nanocomposite beams. Composites Science and Technology 2017;142:235-45.

[30] She GL, Yuan FG, Ren YR, Liu HB, Xiao WS. Nonlinear bending and vibration analysis of functionally graded porous tubes via a nonlocal strain gradient theory. Composite Structures 2018;203:614-23.

[31] She GL, Yuan FG, Karami B, Ren YR, Xiao WS. On nonlinear bending behavior of fg porous curved nanotubes. International Journal of Engineering Science 2019;135:58-74.

[32] Li L, Li X, Hu Y. Nonlinear bending of a two-dimensionally functionally graded beam. Composite Structures 2018;184:1049-61.

[33] Lin J, Li J, Guan Y, Zhao G, Naceur H, Coutellier D. Geometrically nonlinear bending analysis of functionally graded beam with variable thickness by a meshless method. Composite Structures 2018;189:239-46.

[34] Sahmani S, Aghdam MM, Rabczuk T. Nonlinear bending of functionally graded porous micro/nano-beams reinforced with graphene platelets based upon nonlocal strain gradient theory. Composite Structures 2018;186:68-78.

[35] Yang T, Tang Y, Li Q, Yang XD. Nonlinear bending, buckling and vibration of bi-directional functionally graded nanobeams. Composite Structures 2018;204:313-9.

[36] Nguyen DK, Nguyen KV, Gan BS, Alexandrov S, et al. Nonlinear bending of elastoplastic functionally graded ceramicmetal beams subjected to nonuniform distributed loads. Applied Mathematics and Computation 2018;333:443-59.

[37] Khaneh Masjedi P, Ovesy HR. Chebyshev collocation method for static intrinsic equations of geometrically exact beams. International Journal of Solids and Structures 2015;54:183-191.

[38] Khaneh Masjedi P, Ovesy HR. Large deflection analysis of geometrically exact spatial beams under conservative and nonconservative loads using intrinsic equations. Acta Mechanica 2015;226(6):1689-706.

[39] Khaneh Masjedi P, Maheri A. Chebyshev collocation method for the free vibration analysis of geometrically exact beams with fully intrinsic formulation. European Journal of Mechanics-A/Solids 2017;66:329-40.

[40] Sotoudeh Z, Hodges DH. Modeling beams with various boundary conditions using fully intrinsic equations. Journal of Applied Mechanics 2011;78(3):031010.

[41] Schillinger D, Evans JA, Reali A, Scott MA, Hughes TJ. Isogeometric collocation: Cost comparison with galerkin methods and extension to adaptive hierarchical nurbs discretizations. Computer Methods in Applied Mechanics and Engineering $2013 ; 267: 170-232$.

[42] Tornabene F, Fantuzzi N, Ubertini F, Viola E. Strong formulation finite element method based on differential quadrature: a survey. Applied Mechanics Reviews 2015;67(2):020801. 
[43] Washizu K. Variational Methods in Elasticity and Plasticity. 2nd ed.; Oxford: Pergamon Press; 1975.

[44] Kováčik J. Correlation between elastic modulus, shear modulus, poissons ratio and porosity in porous materials. Advanced Engineering Materials 2008;10(3):250.

[45] Gibson LJ, Ashby MF. Cellular solids: structure and properties. Cambridge university press; 1999.

[46] Bîrsan M, Altenbach H, Sadowski T, Eremeyev V, Pietras D. Deformation analysis of functionally graded beams by the direct approach. Composites Part B: Engineering 2012;43(3):1315-28.

[47] Hodges DH. Nonlinear composite beam theory. AIAA, Reston, VA; 2006.

[48] Rao BN, Rao GV. On the large deflection of cantilever beams with end rotational load. ZAMM-Journal of Applied Mathematics and Mechanics/Zeitschrift für Angewandte Mathematik und Mechanik 1986;66(10):507-9.

[49] Argyris JH, Symeonidis S. Nonlinear finite element analysis of elastic systems under nonconservative loading-natural formulation. part i. quasistatic problems. Comput Method Appl M 1981;26(1):75-123.

\section{AppendixA. Beam Kinematics}

A comprehensive description of the kinematics of a spatial geometrically exact beam is presented by Khaneh Masjedi and Ovesy [37]. In order for this work to be self-contained some key relations are reported from Khaneh Masjedi and Ovesy [37] herein.

The position vectors $\mathbf{r}$ and $\mathbf{R}$ which denote the corresponding position of a generic point on the cross section of the beam in the undeformed and deformed state of the beam can be written as:

$$
\begin{gathered}
\mathbf{r}=\mathbf{r}\left(x_{1}, x_{2}, x_{3}\right)=\mathbf{r}_{0}\left(x_{1}\right)+x_{\alpha} \mathbf{e}_{\alpha} \\
\mathbf{R}=\mathbf{R}\left(x_{1}, x_{2}, x_{3}\right)=\mathbf{R}_{0}\left(x_{1}\right)+x_{\alpha} \mathbf{e}_{\alpha}^{*}
\end{gathered}
$$

where $\mathbf{r}_{0}$ and $\mathbf{R}_{0}$ are the position vectors of the beam axis in the undeformed and deformed state of the beam, respectively, $x_{\alpha}$ is the position of a material point on the beam cross-section and $\alpha$ varies from 2 to 3. The displacement vector of the reference line of the beam can be expressed as:

$$
\mathbf{u}_{0}=\mathbf{R}_{0}-\mathbf{r}_{0}
$$

The 1D generalized strain measures for an initially curved and twisted beam can be expressed as:

$$
\begin{gathered}
\boldsymbol{\gamma}=\boldsymbol{\Lambda}^{T} \cdot \mathbf{R}_{0,1}-\mathbf{r}_{0,1} \\
\boldsymbol{\kappa}=\boldsymbol{\Lambda}^{T} . \mathbf{K}-\mathbf{k}
\end{gathered}
$$


where $\boldsymbol{\Lambda}$ is the linear transformation that relates the inertial reference frame and the deformed frame. One can easily obtain the following expressions:

$$
\begin{aligned}
& \mathbf{R}_{0,1}=\left(1+\gamma_{11}\right) \mathbf{e}_{1}^{*}+2 \gamma_{1 \alpha} \mathbf{e}_{\alpha}^{*} \\
& \kappa_{i} \mathbf{e}_{i}^{*}=\left(K_{i}-k_{i}\right) \mathbf{e}_{i}^{*}
\end{aligned}
$$

\section{AppendixB. Chebyshev Polynomials}

The Chebyshev polynomials are a sequence of orthogonal polynomials. The Chebyshev polynomial $T_{n}(x)$ of the first kind is a polynomial in $x$ of degree $n$ and is defined as:

$$
T_{n}(x)=\cos (n \theta) \quad \text { when } \quad x=\cos \theta, \quad-1 \leqslant x \leqslant+1
$$

Combining the trigonometric identity,

$$
\cos (n \theta)+\cos ((n-2) \theta)=2 \cos \theta \cos (n-1) \theta
$$

with Eq.(B.1), the recurrence relation can be obtained as:

$$
\begin{aligned}
& T_{n}(x)=2 x T_{n-1}(x)-T_{n-2}(x), \quad n=2,3, \ldots, \\
& \text { where } \\
& T_{0}(x)=1, T_{1}(x)=x
\end{aligned}
$$

The first few Chebyshev polynomials of the first kind are:

$$
\begin{aligned}
& T_{2}(x)=2 x^{2}-1 \\
& T_{3}(x)=4 x^{3}-3 x \\
& T_{4}(x)=8 x^{4}-8 x^{2}+1 \\
& T_{5}(x)=16 x^{5}-20 x^{3}+5 x
\end{aligned}
$$

Using the weight function $w=\left(1-x^{2}\right)^{-\frac{1}{2}}$ one can find that the Chebyshev polynomials of the first kind satisfy the orthogonality condition:

$$
\int_{-1}^{1} \frac{T_{i}(x) T_{j}(x)}{\sqrt{1-x^{2}}}=\left\{\begin{array}{lr}
0, & i \neq j \\
\frac{\pi}{2}, & i=j \neq 0 \\
\pi, & i=j=0
\end{array}\right.
$$


It is noted that for any arbitrary range $a \leqslant x \leqslant b$ the Chebyshev polynomials can be shifted by replacing the independent variable $x$ in Eq. B.3 by

$$
x=\frac{2}{b-a} x-\frac{b+a}{b-a}
$$

